\section{NICE guidelines on treating schizophrenia - audit}

We recently carried out an audit of clinical practice in our assertive community treatment team, against the National Institute for Health and Clinical Excellence (NICE) clinical guidelines on 'core interventions in the treatment and management of schizophrenia' (NICE, 2002). The guidelines included recommendations that clinical practice should be audited against them and guidance about how to do this.

Our audit aimed to study the compliance with NICE guidelines in a specific team, using direct care-coordinator inquiry and to try to identify reasons for patients not receiving interventions recommended by NICE. Of the assertive community treatment team 84 caseload, 61 were diagnosed with schizophrenia (ICD-10). An eight-item audit tool was used to collect data from care coordinators, using a structured interview method.

We found variable levels of compliance, ranging from $0 \%$ for production of advance directive to $85 \%$ for the provision of written information to service users/carers. Where interventions were not carried out, this often appeared understandable. In the example of family therapy, only $20 \%$ had received formal approach but of the remainder $20 \%$ declined, $15 \%$ had other forms of family/ care support, $51 \%$ were not in contact with their family and $8 \%$ had no clinical indication. Similarly, of the 21 cases where cognitive-behavioural therapy had not been offered, 11 were unable to make an informed choice and 3 had no persisting symptoms. Non-compliance was often associated with problems which seemed understandable and perhaps insurmountable. This raises questions about the utility of NICE guidelines as a means to guide best practice, and suggests that compliance with guidelines needs to be considered carefully in the context of the overall treatment plan.

The findings relating to cognitivebehavioural therapy and antipsychotic polypharmacy were interesting: several patients appeared to be choosing to continue to take more than one antipsychotic or not to have the therapy. This raises a dilemma, which seems common clinically, of how to promote best practice while also being mindful of patient choice and empowerment.

Although no patients had advance directives, 70\% had crisis/'rainy day' plans. It is our view that explicit managerial prioritisation would be needed to bring in a routine use of advance directives, and this is unlikely to occur. As Dopson et al (2001) point out, the key factors in implementing clinical guidelines are a strong evidence base, supportive opinion leaders and integration of the use of a guideline approach within an organisation.

Our perception is that the link between clinical interventions and outcome, particularly in the treatment of mental disorders, is not straightforward. Dent \& Sadler (2002) rightly note that professionals perceive ambiguity about how NICE reaches its conclusions, the lack of consistent support for implementation and uncertainty about who should monitor compliance.

We found the results of the audit interesting and it has enabled a form of reflective practice at the team level. We would be interested to hear from other teams that have gone through a similar process, and recommend the method used as a simple way to benchmark a team's work and consider training needs. Further audit and research into the impact of guidelines on meeting the needs of individuals with severe mental illness is needed.

DENT,T. H. \& SADLER, M. (2002) From guidance to practice: why NICE is not enough. BMJ, 324, 842845.

DOPSON, S., LOCOCK, L., CAMBERS, D., et al (2001) Implementation of evidence-based medicine: evaluation of the Promoting Action on Clinical Effectiveness programme. Journal of Health Services Research and Policy, 187, 248-255.

NATIONAL INSTITUTE FOR CLINICAL EXCELLENCE (2002) Core Interventions in the Treatment and Management of Schizophrenia in Primary and Secondary Care. http://www.nice.org.uk/guidance/ index.jsp?action $=$ bylD\&o=10916

*Rob Macpherson Consultant Rehabilitation Psychiatrist, Wotton Lawn, Horton Rd, Gloucester GL13WL, email: rob.macpherson@glos.nhs.uk, Nicola Hovey Audit Manager, Gloucestershire Partnership NHS Trust, Rikenell, Montpellier,

Gloucester, Krishen Ranganath Specialist Registrar in Psychiatry, Amjad Uppal Specialist Registrar in Psychiatry, Wotton lawn, Gloucester,

AndyThompson Consultant Psychiatrist, Orygen Youth Health, Melbourne, Australia

doi: 10.1192/pb.32.2.75

\section{Highlighting a neglected component of recovery}

Schrank \& Slade's article on recovery in psychiatry (Psychiatric Bulletin, September 31, 321-325) is important for three reasons. First, it provides an exhaustive coverage. Second, many components contributing to recovery are also nonspecific therapeutic factors, like: (a) accepting the illness; (b) hope; (c) selfconfidence; (d) courage, including an attitudinal readiness to experiment with tolerable risks; (e) responsibility and control, encompassing 'internal locus of control'; (f) recognising one's values, strengths and limitations, which includes self-monitoring and evaluation. Similar factors also constitute desirable therapist qualities. Third, and the most important, is that the authors have resurrected the age-old concept of 'determination'.

I believe that this concept is closely related to that of 'will', which is probably even more fundamental as regards mental health recovery. Determination also shares some common components with psychological phenomena placebo effect, motivation and expectation, and it plays an important role in clinical outcomes, for example in cancer survival.

The concept of will (as in 'will power') on the other hand, is deeply embedded in all human cultures. A 'will to survive' has appeared in war and heroic anecdotes throughout centuries and a 'will-to-win' has been the mantra in competitive sports. Even though this concept is difficult to operationally define, it is unquestionably worthy of investigation by the mental health profession.

Channapatna Shamasundar Consultant Psychiatrist, Bangalore, India, email: drshamasundar @hotmail.com

doi: $10.1192 / p b .32 .2 .75 a$

\section{A Devil's advocate}

Whelan et al's article on Medical Training Application System (MTAS) fiasco (Psychiatric Bulletin, November 2007, 31, 425-427) reincarnates the proverbial dead horse. Was there another side to the story? Why do the Colleges tend to forget that they had been consulted on the process before it was implemented? Our College organised meetings on MTAS in London and then pulled out senior house officers from the farthest corners of the UK to sell it. Did they raise a brow regarding the questions on the application form?

But more importantly, was it wise to have changed horses mid-stream? As someone who went through MTAS' birth pangs, I can say that the panic started when 'surprisingly' many were not shortlisted. Forgotten was the lost tribe who had been on the list and as we went through the interview process, we found that most of those who were shortlisted had more than two interviews. Hypothesis: was the system more specific than sensitive, as all screening processes ought to be? Had the process been allowed to run through to its original programme, the second round 
columns would have seen many vacancies, as those who had been through the first round would have taken only one runthrough slot despite being offered many interviews. Hypothesis: could then the system have balanced it, as second round vacancies would be proportionate to the candidates unsuccessful in the first round?

Were we too unnerved to give the system a fair trial as designed (now conspiracy!!) originally? Would any process of selection in future be credible not only in finding a doctor, but distinguishing a surgeon's glove from a psychiatrist's couch?

Vellingiri Raja Badrakalimuthu Specialty Registrar, Drug and Alcohol Services, Norfolk and Waveney Mental Health Partnership NHS Trust, Great Yarmouth, email: dr_vellingiriraja@yahoo.co.uk doi: $10.1192 / p b .32 .2 .75 b$ 